\title{
Generalizations of the Sommerfield and Schwinger models
}

\section{Howard Georgi and Brian Warner}

Center for the Fundamental Laws of Nature, Jefferson Physical Laboratory, Harvard University, Cambridge, MA 02138, U.S.A.

E-mail: hgeorgi@fas.harvard.edu, brian_warner@college.harvard.edu

ABstract: The Sommerfield model with a massive vector field coupled to a massless fermion in $1+1$ dimensions is an exactly solvable analog of a Bank-Zaks model. The "physics" of the model comprises a massive boson and an unparticle sector that survives at low energy as a conformal field theory (Thirring model). We analyze generalizations of the Sommerfield model, and the corresponding generalizations of the Schwinger model, with more massless fermions and more vector fields.

Keywords: Field Theories in Lower Dimensions, Conformal Field Theory

ArXiv EPrint: 1907.12705 


\section{Contents}

1 Generalized Sommerfield model of a Banks-Zaks sector 1

$2 n_{F}$ fermions $\quad 3$

$\begin{array}{lll}3 & n_{A} \text { vectors } & 5\end{array}$

$\begin{array}{llll}4 & n_{A} \text { vectors and } n_{F} \text { fermions } & 8\end{array}$

5 Correlation functions 10

6 Conclusions 11

\section{Generalized Sommerfield model of a Banks-Zaks sector}

The study of explicitly solvable field theories in $1+1$ dimensions has been a fruitful source of important ideas about quantum field theories in general [1-3]. In this note, we hope to contribute to this rich legacy by constructing and analyzing generalizations of the Schwinger and Sommerfield [4] models with multiple massless fermions and vector fields. ${ }^{1}$ In this note, we find the general fermion correlation functions for an arbitrary number of massless fermions with commuting couplings to an arbitrary number vector fields in $1+1$ dimensions. While the general correlators including the vector fields are all calculable using the results we find in this paper, we focus on the fermion correlators which are particularly interesting because they determine the long-distance physics. A number of interesting special cases have appeared in the literature, see for example [6, 7], and [8].

In [9], one of us suggested that it useful to think of the Schwinger model as a limit of the Sommerfield model as the gauge bare vector meson mass goes to zero. In a separate note [10], we will use the technology developed in this paper to construct and analyze a number of generalized Schwinger models.

\footnotetext{
${ }^{1}$ Our conventions, as in [5], are: $g^{00}=-g^{11}=1, \epsilon^{01}=-\epsilon^{10}=-\epsilon_{01}=\epsilon_{10}=1$. From the defining properties $\left\{\gamma^{\mu}, \gamma^{\nu}\right\}=2 g^{\mu \nu}$ and $\gamma^{5}=-\frac{1}{2} \epsilon_{\mu \nu} \gamma^{\mu} \gamma^{\nu}$, it follows that $\gamma^{\mu} \gamma^{5}=-\epsilon^{\mu \nu} \gamma_{\nu}$ and $\gamma^{\mu} \gamma^{\nu}=g^{\mu \nu}+\epsilon^{\mu \nu} \gamma^{5}$, and we will use the representation $\gamma^{0}=\left(\begin{array}{ll}0 & 1 \\ 1 & 0\end{array}\right), \gamma^{1}=\left(\begin{array}{cc}0 & -1 \\ 1 & 0\end{array}\right), \gamma^{5}=\gamma^{0} \gamma^{1}=\left(\begin{array}{cc}1 & 0 \\ 0 & -1\end{array}\right)$. Then the components $\psi_{1}$ and $\psi_{2}$ describe right-moving and left-moving fermions, respectively. Lightcone coordinates are defined by

$$
\begin{array}{rlrl}
x^{ \pm} & =x^{0} \pm x^{1} & \partial_{ \pm}=\frac{\partial_{0} \pm \partial_{1}}{2} & \\
x^{+} \partial_{+}+x^{-} \partial_{-} & =\left(x^{0}+x_{1}\right) \frac{\partial_{0}+\partial_{1}}{2}+\left(x^{0}-x_{1}\right) \frac{\partial_{0}-\partial_{1}}{2}=x^{0} \partial_{0}+x^{1} \partial_{1} & \\
A^{0} & =\partial^{0} \mathcal{V} / m_{0}-\partial^{1} \mathcal{A} / m & A^{1} & =\partial^{1} \mathcal{V} / m_{0}-\partial^{0} \mathcal{A} / m \\
A_{0} & =\partial_{0} \mathcal{V} / m_{0}+\partial_{1} \mathcal{A} / m & A_{1} & =\partial_{1} \mathcal{V} / m_{0}+\partial_{0} \mathcal{A} / m \quad A_{ \pm}=\partial_{ \pm} \mathcal{V} / m_{0} \pm \partial_{ \pm} \mathcal{A} / m
\end{array}
$$
}


We will begin with a quick review of the Sommerfield model to set notation. ${ }^{2}$ The Sommerfield Lagrangian is

$$
\mathcal{L}_{S}=\bar{\psi}(i \not \supset-e \not A) \psi-\frac{1}{4} F^{\mu \nu} F_{\mu \nu}+\frac{m_{0}^{2}}{2} A^{\mu} A_{\mu}
$$

To solve the model, we decompose $A^{\mu}$ as

$$
A^{\mu}=\partial^{\mu} \mathcal{V} / m_{0}+\epsilon^{\mu \nu} \partial_{\nu} \mathcal{A} / m
$$

where

$$
m^{2}=m_{0}^{2}+e^{2} / \pi
$$

Then we can write

$$
\epsilon_{\mu \nu} \partial^{\mu} A^{\nu}=\epsilon_{\mu \nu} \partial^{\mu} \epsilon^{\nu \beta} \partial_{\beta} \mathcal{A} / m=\partial_{\mu} \partial^{\mu} \mathcal{A} / m \quad \partial_{\mu} A^{\mu}=\partial_{\mu} \partial^{\mu} \mathcal{V} / m_{0}
$$

and the Lagrangian becomes

$$
\begin{aligned}
\mathcal{L}_{S}= & i \bar{\psi} \not \partial \psi-e \bar{\psi} \gamma_{\mu} \psi\left(\partial^{\mu} \mathcal{V} / m_{0}+\epsilon^{\mu \nu} \partial_{\nu} \mathcal{A} / m\right) \\
& +\frac{1}{2 m^{2}} \mathcal{A} \square^{2} \mathcal{A}+\frac{1}{2} \partial_{\mu} \mathcal{V} \partial^{\mu} \mathcal{V}-\frac{m_{0}^{2}}{2 m^{2}} \partial_{\mu} \mathcal{A} \partial^{\mu} \mathcal{A}
\end{aligned}
$$

If we change the fermionic variable to

$$
\Psi=e^{i e\left(\mathcal{V} / m_{0}+\mathcal{A} \gamma^{5} / m\right)} \psi
$$

the fermion becomes free:

$$
\mathcal{L}_{S}=i \bar{\Psi} \not \partial \Psi+\frac{1}{2} \partial_{\mu} \mathcal{V} \partial^{\mu} \mathcal{V}+\frac{1}{2 m^{2}} \mathcal{A} \square^{2} \mathcal{A}-\frac{1}{2} \partial_{\mu} \mathcal{A} \partial^{\mu} \mathcal{A}
$$

While the final result is expressed in terms of free fields, it is still worth being careful about the definitions of composite operators, with several local fields at the same point. Because of the super-renormalizable interactions, it is not much of an issue in these theories. Almost all the relevant operators can be defined simply as the leading operators in an operator product expansion [12]. However, there is one important exception. In the last terms in (1.7), $m_{0}^{2} / \mathrm{m}^{2}$ has been replaced by 1 in order to account for the fact that the path integral measure is not invariant under the $\mathcal{A}$ part of (1.6) [13]. ${ }^{3}$ This effect can be seen in many different ways.

It is worth recalling how this works in more detail. We can get from the kinetic energy in (1.5)

$$
\bar{\psi} \gamma_{\mu}\left(i \partial^{\mu}-e\left(\partial^{\mu} \mathcal{V} / m_{0}+\epsilon^{\mu \nu} \partial_{\nu} \mathcal{A} / m\right)\right) \psi
$$

to

$$
\overline{e^{i e\left(\mathcal{V} / m_{0}\right)} \psi} \gamma_{\mu}\left(i \partial^{\mu}-e \epsilon^{\mu \nu} \partial_{\nu} \mathcal{A} / m\right) e^{i e\left(\mathcal{V} / m_{0}\right)} \psi
$$

\footnotetext{
${ }^{2}$ The analysis is similar to that in [11] and inspired by the treatment of the Schwinger model in [6].

${ }^{3}$ The same effect gives mass $e / \sqrt{\pi}$ to the gauge boson in the Schwinger model. See also [14].
} 
by an ordinary vector gauge transformation, so (1.8) and (1.9) are equal. But $\partial^{\mu} \mathcal{A}$ has axial-vector couplings because

$$
\gamma_{\mu} \epsilon^{\mu \nu} \partial_{\nu} \mathcal{A}=\gamma_{\mu} \gamma^{5} \partial^{\mu} \mathcal{A}
$$

and an axial gauge transformation induces a change in the Lagrangian. The effect from an infintesimal axial transformation is proportional to the 2D axial anomaly,

$$
\begin{aligned}
\partial_{\mu} j_{5}^{\mu}=-\frac{e}{\pi} \epsilon^{\mu \nu} \partial_{\mu} A_{\nu} & =-\frac{e}{\pi} \square \mathcal{A} \\
\frac{d}{d \alpha} \overline{e^{i e\left(\mathcal{V} / m_{0}+\alpha \mathcal{A} \gamma^{5} / m\right)}} \psi & \gamma_{\mu}\left(i \partial^{\mu}-e \epsilon^{\mu \nu} \partial_{\nu}(1-\alpha) \mathcal{A} / m\right) e^{i e\left(\mathcal{V} / m_{0}+\alpha \mathcal{A} \gamma^{5} / m\right)} \psi \\
& =-\frac{e^{2}}{m^{2} \pi} \mathcal{A}(1-\alpha) \square \mathcal{A}
\end{aligned}
$$

Integrating (1.12) from $\alpha=0$ to 1 gives

$$
\begin{aligned}
\overline{e^{i e \mathcal{V} / m_{0}} \psi} \gamma_{\mu}\left(i \partial^{\mu}-e \epsilon^{\mu \nu} \partial_{\nu} \mathcal{A} / m\right) e^{i e \mathcal{V} / m_{0}} \psi & =i \bar{\Psi} \not \supset \Psi+\frac{e^{2}}{2 m^{2} \pi} \mathcal{A} \square \mathcal{A} \\
& =i \bar{\Psi} \not \supset \Psi-\frac{e^{2}}{2 m^{2} \pi} \partial_{\mu} \mathcal{A} \partial^{\mu} \mathcal{A}
\end{aligned}
$$

where $\Psi$ is given by (1.6). The second term in (1.13) produces the change in the coefficient of the $\mathcal{A}$ kinetic energy to its canonical value.

Focusing on $\mathcal{A}$ in (1.7), we can replace it with somewhat more normal looking fields as follows.

$$
\begin{aligned}
\frac{1}{2 m^{2}} \mathcal{A} \square^{2} \mathcal{A}-\frac{1}{2} \partial_{\mu} \mathcal{A} \partial^{\mu} \mathcal{A} & \rightarrow-\frac{m^{2}}{2} \mathcal{B}^{2}+\mathcal{B} \square \mathcal{A}-\frac{1}{2} \partial_{\mu} \mathcal{A} \partial^{\mu} \mathcal{A} \\
& =-\frac{m^{2}}{2} \mathcal{B}^{2}+\frac{1}{2} \partial_{\mu} \mathcal{B} \partial^{\mu} \mathcal{B}-\frac{1}{2} \partial_{\mu} \mathcal{C} \partial^{\mu} \mathcal{C}
\end{aligned}
$$

where $\mathcal{C}=\mathcal{A}+\mathcal{B}$, so $\mathcal{B}$ is a massive field and $\mathcal{C}$ is a massless ghost and the Lagrangian becomes

$$
\mathcal{L}_{S}=i \bar{\Psi} \not \partial \Psi+\frac{1}{2} \partial_{\mu} \mathcal{V} \partial^{\mu} \mathcal{V}-\frac{m^{2}}{2} \mathcal{B}^{2}+\frac{1}{2} \partial_{\mu} \mathcal{B} \partial^{\mu} \mathcal{B}-\frac{1}{2} \partial_{\mu} \mathcal{C} \partial^{\mu} \mathcal{C}
$$

and the original fermion and vector fields can be written in terms of free fields

$$
\begin{aligned}
& \psi_{S}=e^{-i e\left(\mathcal{V} / m_{0}+(\mathcal{C}-\mathcal{B}) \gamma^{5} / m\right)} \Psi \\
& A_{S}^{\mu}=\partial^{\mu} \mathcal{V} / m_{0}+\epsilon^{\mu \nu} \partial_{\nu}(\mathcal{C}-\mathcal{B}) / m
\end{aligned}
$$

In the following sections, we generalize this analysis to include more fermions and more vector bosons. We can then use the generalizations of (1.17) and (1.18) to write down the Green's functions as in [11]. This is done in section 5.

\section{$2 \quad n_{F}$ fermions}

We now need an index, $\alpha=1$ to $n_{F}$ for the fermions, and we can write the Lagrangian as

$$
\mathcal{L}_{f}=\left(\sum_{\alpha=1}^{n_{F}} \bar{\psi}_{\alpha}\left(i \not \partial-[e]_{\alpha} \not A\right) \psi_{\alpha}\right)-\frac{1}{4} F^{\mu \nu} F_{\mu \nu}+\frac{m_{0}^{2}}{2} A^{\mu} A_{\mu}
$$


We have written the coupling in a peculiar way because in further generalizations, it will be convenient to use a matrix notation and think of the couplings as forming a vector, $e$, in the fermion space, so $[e]_{\alpha}$ is just the $\alpha$ component of $e$. Again, we decompose $A^{\mu}$ as

$$
A^{\mu}=\partial^{\mu} \mathcal{V} / m_{0}+\epsilon^{\mu \nu} \partial_{\nu} \mathcal{A} / m
$$

where

$$
m^{2}=m_{0}^{2}+e e^{T} / \pi
$$

because each of the massless fermions contributes to dynamical mass of the vector field. Thus (2.1) becomes

$$
\begin{aligned}
\mathcal{L}_{f}= & \left(\sum_{\alpha=1}^{n_{F}}\left(i \bar{\psi}_{\alpha} \not \partial \psi_{\alpha}-e_{\alpha} \bar{\psi}_{\alpha} \gamma_{\mu} \psi_{\alpha}\left(\partial^{\mu} \mathcal{V} / m_{0}+\epsilon^{\mu \nu} \partial_{\nu} \mathcal{A} / m\right)\right)\right) \\
& +\frac{1}{2 m^{2}} \mathcal{A} \square^{2} \mathcal{A}+\frac{1}{2} \partial_{\mu} \mathcal{V} \partial^{\mu} \mathcal{V}-\frac{m_{0}^{2}}{2 m^{2}} \partial_{\mu} \mathcal{A} \partial^{\mu} \mathcal{A}
\end{aligned}
$$

If we change the fermionic variables to

$$
\Psi_{\alpha}=e^{i[e]_{\alpha}\left(\mathcal{V} / m_{0}+\mathcal{A} \gamma^{5} / m\right)} \psi
$$

the fermions becomes free:

$$
\mathcal{L}_{f}=\left(\sum_{\alpha=1}^{n_{F}} i \bar{\Psi}_{\alpha} \not \partial \Psi_{\alpha}\right)+\frac{1}{2} \partial_{\mu} \mathcal{V} \partial^{\mu} \mathcal{V}+\frac{1}{2 m^{2}} \mathcal{A} \square^{2} \mathcal{A}-\frac{1}{2} \partial_{\mu} \mathcal{A} \partial^{\mu} \mathcal{A}
$$

As usual, in the last terms in (2.6) $m_{0}^{2} / \mathrm{m}^{2}$ has been replaced by 1 in order to account for the fact that the path integral measure is not invariant under the $\mathcal{A}$ part of (2.5) [13].

Again we can replace $\mathcal{A}$ in (2.6) with somewhat more normal looking fields as follows.

$$
\begin{aligned}
\frac{1}{2 m^{2}} \mathcal{A} \square^{2} \mathcal{A}-\frac{1}{2} \partial_{\mu} \mathcal{A} \partial^{\mu} \mathcal{A} & \rightarrow-\frac{m^{2}}{2} \mathcal{B}^{2}+\mathcal{B} \square \mathcal{A}-\frac{1}{2} \partial_{\mu} \mathcal{A} \partial^{\mu} \mathcal{A} \\
& =-\frac{m^{2}}{2} \mathcal{B}^{2}+\frac{1}{2} \partial_{\mu} \mathcal{B} \partial^{\mu} \mathcal{B}-\frac{1}{2} \partial_{\mu} \mathcal{C} \partial^{\mu} \mathcal{C}
\end{aligned}
$$

where $\mathcal{C}=\mathcal{A}+\mathcal{B}$, so $\mathcal{B}$ is a massive field and $\mathcal{C}$ is a massless ghost. Then

$$
\mathcal{L}_{f}=\left(\sum_{\alpha=1}^{n_{F}} i \bar{\Psi}_{\alpha} \not \partial \Psi_{\alpha}\right)+\frac{1}{2} \partial_{\mu} \mathcal{V} \partial^{\mu} \mathcal{V}-\frac{m^{2}}{2} \mathcal{B}^{2}+\frac{1}{2} \partial_{\mu} \mathcal{B} \partial^{\mu} \mathcal{B}-\frac{1}{2} \partial_{\mu} \mathcal{C} \partial^{\mu} \mathcal{C}
$$

and the original fermion and vector fields can be written in terms of free fields

$$
\psi_{\alpha}=e^{-i[e]_{\alpha}\left(\mathcal{V} / m_{0}+(\mathcal{C}-\mathcal{B}) \gamma^{5} / m\right)} \Psi_{\alpha}
$$

and as usual

$$
A^{\mu}=\partial^{\mu} \mathcal{V} / m_{0}+\epsilon^{\mu \nu} \partial_{\nu}(\mathcal{C}-\mathcal{B}) / m
$$

As usual, we can use (2.10) and (2.11) straightforwardly to write down the Green's functions as in [11]. The long distance physics of (2.9) is a conformal field theory and the fermion fields are conformal operators. 


\section{$3 \quad n_{A}$ vectors}

The analysis also generalizes in a straightforward way to more vector fields. Our generalized Lagrangian is

$$
\mathcal{L}_{A}=\bar{\psi}\left(i \not \partial-e^{T} \not A\right) \psi-\frac{1}{4} F^{T \mu \nu} \cdot F_{\mu \nu}+\frac{1}{2} A^{\mu T} M_{0}^{2} A_{\mu}
$$

where our vector fields $A$ and couplings $e$ are $n_{A}$ dimensional column vectors, and $M_{0}^{2}$ is a positive $n_{A} \times n_{A}$ matrix. ${ }^{4}$ Where it does not cause confusion, we may drop the transposes and not distinguish between row vectors and column vectors, so we can write

$$
\mathcal{L}_{A}=\bar{\psi}(i \not \partial-e \cdot \not A) \psi-\frac{1}{4} F^{\mu \nu} \cdot F_{\mu \nu}+\frac{1}{2} A^{\mu} M_{0}^{2} A_{\mu}
$$

As in the Sommerfield model, to solve the model, it is convenient to decompose $A^{\mu}$ into scalar and pseudo-scalar fields. Because the mass structure is now more complicated, we will do this in two steps. Begin with

$$
A^{\mu}=\partial^{\mu} \mathbb{V}+\epsilon^{\mu \nu} \partial_{\nu} \mathbb{A}
$$

The Lagrangian becomes

$$
\begin{aligned}
\mathcal{L}= & i \bar{\psi} \not \partial \psi-\bar{\psi} \gamma_{\mu} \psi e \cdot\left(\partial^{\mu} \mathbb{V}+\epsilon^{\mu \nu} \partial_{\nu} \mathbb{A}\right)+\frac{1}{2} \mathbb{A} \cdot \square^{2} \mathbb{A} \\
& +\frac{1}{2}\left(\partial_{\mu} \mathbb{V} M_{0}^{2} \partial^{\mu} \mathbb{V}-\partial_{\mu} \mathbb{A} M_{0}^{2} \partial^{\mu} \mathbb{A}\right)
\end{aligned}
$$

If we change the fermionic variable to

$$
\Psi=e^{i e \cdot\left(\mathbb{V}+\mathbb{A} \gamma^{5}\right)} \psi
$$

the fermion becomes free:

$$
\mathcal{L}=i \bar{\Psi} \not \partial \Psi+\frac{1}{2} \partial_{\mu} \mathbb{V} M_{0}^{2} \partial^{\mu} \mathbb{V}+\frac{1}{2} \mathbb{A} \cdot \square^{2} \mathbb{A}-\frac{1}{2} \partial_{\mu} \mathbb{A} M^{2} \partial^{\mu} \mathbb{A}
$$

In the last term of (3.6) $M_{0}^{2}$ has been replaced by

$$
M^{2}=M_{0}^{2}+\frac{1}{\pi} e e^{T}
$$

This is the same physics that we saw in (1.7) in order to account for the fact that the path integral measure is not invariant under the $\mathcal{A}$ part of (3.9) [13].

So far, we are considering the case in which both $M_{0}^{2}$ and $M^{2}$ are positve matrices, so we can define the positive square roots $M_{0}$ and $M$ and use these to simplify things by taking

$$
\mathcal{V}=M_{0} \mathbb{V} \text { and } \mathcal{A}=M \mathbb{A}
$$

\footnotetext{
${ }^{4}$ Later, we will relax this condition and consider what happens when $m_{0}^{2}$ has a zero eigenvalue, but we can approach this interesting case as a limit.
} 
and write the original $\psi$ and $A^{\mu}$ fields in the Lagrangian in terms of free fields, as in the Sommerfield model.

$$
\begin{gathered}
\psi=e^{-i e \cdot\left(M_{0}^{-1} \mathcal{V}+M^{-1} \mathcal{A} \gamma^{5}\right)} \Psi \\
A^{\mu}=M_{0}^{-1} \partial^{\mu} \mathcal{V}+M^{-1} \epsilon^{\mu \nu} \partial_{\nu} \mathcal{A}
\end{gathered}
$$

Now the $\mathcal{V}$ kinetic energy is conventional and as in section 3, we can replace $\mathcal{A}$ with more normal-looking fields -

$$
\begin{aligned}
\frac{1}{2} \mathcal{A} M^{-2} \square^{2} \mathcal{A}-\frac{1}{2} \partial_{\mu} \mathcal{A} \cdot \partial^{\mu} \mathcal{A} & \rightarrow-\frac{1}{2} \mathcal{B} M^{2} \mathcal{B}+\mathcal{B} \cdot \square \mathcal{A}-\frac{1}{2} \partial_{\mu} \mathcal{A} \cdot \partial^{\mu} \mathcal{A} \\
& =-\frac{1}{2} \mathcal{B} M^{2} \mathcal{B}+\frac{1}{2} \partial_{\mu} \mathcal{B} \cdot \partial^{\mu} \mathcal{B}-\frac{1}{2} \partial_{\mu} \mathcal{C} \cdot \partial^{\mu} \mathcal{C}
\end{aligned}
$$

where $\mathcal{C}=\mathcal{A}+\mathcal{B}$. Then $\mathcal{B}$ are massive fields and $\mathcal{C}$ are massless ghosts. And

$$
\begin{aligned}
\psi & =e^{-i e \cdot\left(M_{0}^{-1} \mathcal{V}+M^{-1}(\mathcal{C}-\mathcal{B}) \gamma^{5}\right)} \Psi \\
A^{\mu} & =M_{0}^{-1} \partial^{\mu} \mathcal{V}+M^{-1} \epsilon^{\mu \nu} \partial_{\nu}(\mathcal{C}-\mathcal{B})
\end{aligned}
$$

We are free to make an orthogonal transformation on the vector fields which induces an orthogonal transformation on the $e$ matrix,

$$
e \rightarrow O e
$$

where $O$ is a real orthogonal $n_{A} \times n_{A}$ matrix. We can use this freedom to take the physical vector boson mass matrix $M$ to be diagonal and write

$$
[M]_{j k}=m_{j} \delta_{j k} \quad\left[M_{0}^{2}\right]_{j k}=m_{j}^{2} \delta_{j k}-\frac{[e]_{j}[e]_{k}}{\pi}
$$

Then the couplings of the $\mathcal{B}$ and $\mathcal{C}$ are simple, and all the complication is in the couplings of the $\mathcal{V}$.

$$
\begin{aligned}
\psi & =e^{-i e \cdot\left(M_{0}^{-1} \mathcal{V}+M^{-1}(\mathcal{C}-\mathcal{B}) \gamma^{5}\right)} \Psi=e^{-i\left(e \cdot M_{0}^{-1} \mathcal{V}+\sum_{j}\left(e_{j} / m_{j}\right)\left([\mathcal{C}]_{j}-[\mathcal{B}]_{j}\right) \gamma^{5}\right)} \Psi \\
{\left[A^{\mu}\right]_{j} } & =\left[M_{0}^{-1} \partial^{\mu} \mathcal{V}\right]_{j}+\frac{1}{m_{j}} \epsilon^{\mu \nu} \partial_{\nu}\left([\mathcal{C}]_{j}-[\mathcal{B}]_{j}\right)
\end{aligned}
$$

Notice that (3.15) and the positivity of $M_{0}^{2}$ implies that if $m_{j}=0$, then $[e]_{j}$ must be zero as well, so $A_{j}$ decouples and is not interesting. So we are not interested in the situtation in which any of the $m_{j}$ go to zero and will assume that they are all positive.

We can use (3.15) to simplify the calculation of the $M_{0}$ dependence of the correlators. Note that (3.15) can be written as

$$
M_{0}^{2}=M^{2}-\frac{1}{\pi} e e^{T}=M\left(I-\frac{1}{\pi} M^{-1} e e^{T} M^{-1}\right) M
$$

This is positive so long as the eigenvalues of the matrix

$$
\tilde{B}_{1} \equiv \frac{1}{\pi} M^{-1} e e^{T} M^{-1}
$$


are less than 1 . The matrix $\tilde{B}_{1}$ is rank-1 and all but one of the eigenvalues vanish, so the non-zero eigenvalue is just the trace

$$
\beta=\operatorname{tr} \tilde{B}_{1}=\frac{1}{\pi} e^{T} M^{-2} e=\frac{1}{\pi} \sum_{j} \frac{[e]_{j}^{2}}{m_{j}^{2}}
$$

Thus we lose positivity of $M_{0}^{2}$ if and only if $\beta>1$ so we must have

$$
\sum_{j} \frac{e_{j}^{2}}{m_{j}^{2}} \leq \pi
$$

The equality corresponds to the interesting case in which one of the eigenvalues of $M_{0}$ goes to zero. This is the "Schwinger point" [9] at which one linear combination of the vectors has zero mass so there is a gauge invariance. We will discuss that further in [10].

But going on, we can now formally write down $M_{0}^{-2}$, in the form

$$
M_{0}^{-2}=M^{-1}\left(I+\frac{\gamma}{\pi} M^{-1} e e^{T} M^{-1}\right) M^{-1}
$$

Then $\gamma$ must satisfy

$$
M_{0}^{-2} M_{0}^{2}=I=M^{-1}\left(I+\frac{-1+\gamma-\beta \gamma}{\pi} M^{-1} e e^{T} M^{-1}\right) M
$$

so

$$
\gamma=\frac{1}{1-\beta}
$$

and

$$
M_{0}^{-2}=M^{-1}\left(I+\frac{1}{\pi(1-\beta)} M^{-1} e e^{T} M^{-1}\right) M^{-1}
$$

In the fermion correlators, from (3.16), because the $\mathcal{V}$ are all massless, the mass matrix $M_{0}$ only appears in the combination $e^{T} M_{0}^{-2} e$ which from (3.25) is

$$
e^{T} M_{0}^{-2} e=e^{T} M^{-1}\left(I+\frac{1}{\pi(1-\beta)} M^{-1} e e^{T} M^{-1}\right) M^{-1} e=\frac{\pi \beta}{1-\beta}
$$

We focus in this note on the fermion correlators, but it is amusing to note that to calculate the correlations functions involving fermions and a single $A_{j}$, the only additional thing we need to calculate that depends on $M_{0}$ is $M_{0}^{-2} e$. A factor of $M_{0}^{-1} e$ comes from the $\mathcal{V}$ dependence of the fermion field - and this is complicated. But this gets multiplied by an additional factor of $M_{0}^{-1}$ from the $A_{j}$ field, and the combination is simple:

$$
M_{0}^{-2} e=M^{-1}\left(I+\frac{1}{\pi(1-\beta)} M^{-1} e e^{T} M^{-1}\right) M^{-1} e=\frac{1}{1-\beta} M^{-2} e
$$

The ghost contribution is just proportional, with the coefficient

$$
e^{T} M^{-2} e=\pi \beta
$$

This is the combination that appears in the unparticle part of the $C$ functions. At the Schwinger point, it just goes to $\pi$. 


\section{$4 \quad n_{A}$ vectors and $n_{F}$ fermions}

This is the general case, so in this section, we have an index for the fermions, $\psi_{\alpha}$ for $\alpha=1$ to $n_{F}$, an index for the vector bosons, $A_{j}^{\mu}$ for $j=1$ to $n_{A}$, and $n_{F}$ sets of couplings, one to each of the fermions. So in a generalization of the notation of the previous section, we can write the couplings as a $n_{F} \times n_{A}$ matrix, $e$, in which matrix in which

$$
[e]_{j \alpha} \text { is the coupling of the } j \text { th vector to the } \alpha \text { th fermion. }
$$

Where the $|j\rangle$ are an orthonormal basis in the index space of the vector fields. We will often think of $e$ as a and use a matrix notation. The Lagrangian is

$$
\mathcal{L}_{A f}=\left(\sum_{\alpha=1}^{n_{F}} \bar{\psi}_{\alpha}\left(i \not \partial-\left[e^{T} \not A\right]_{\alpha}\right) \psi_{\alpha}\right)-\frac{1}{4} F^{\mu \nu} \cdot F_{\mu \nu}+\frac{1}{2} A^{\mu} M_{0}^{2} A_{\mu}
$$

Note that we have assumed that the gauge couplings are diagonal in the fermion space. This is important to ensure that the model is exactly solvable. We could have non-diagonal couplings as long as the couplings to different gauge bosons commute with one another. But then we can simultaneously diagonalize them with a unitary transformation on the fermion fields, so we may as well assume that the gauge couplings are diagonal from the beginning.

As one might expect, the analysis of this model uses a combination of the tools discussed in the two previous sections. Each of the massless fermions generates a contribution to the vector boson mass matrix like that in (3.7),

$$
M^{2}=M_{0}^{2}+\frac{1}{\pi} e e^{T}
$$

As in section 3, we will use our freedom to redefine the vector fields to diagonalize the physical vector boson mass, so as in (3.15), we can write and write

$$
[M]_{j k}=m_{j} \delta_{j k} \quad\left[M_{0}^{2}\right]_{j k}=m_{j}^{2} \delta_{j k}-\sum_{\alpha} \frac{[e]_{j \alpha}[e]_{k \alpha}}{\pi}
$$

Then as in section 3 , the couplings of the $\mathcal{B}$ and $\mathcal{C}$ are simple, and all the complication is in the couplings of the $\mathcal{V}$.

$$
\begin{aligned}
\psi_{\alpha} & =e^{-i\left(\left[e^{T} \cdot M_{0}^{-1} \mathcal{V}\right]_{\alpha}+\left[e^{T} M^{-1}(\mathcal{C}-\mathcal{B})\right]_{\alpha} \gamma^{5}\right)} \Psi_{\alpha} \\
& =e^{-i\left(\left[e^{T} \cdot M_{0}^{-1} \mathcal{V}\right]_{\alpha}+\sum_{j}\left([e]_{j \alpha} / m_{j}\right)\left([\mathcal{C}]_{j}-[\mathcal{B}]_{j}\right) \gamma^{5}\right)} \Psi_{\alpha} \\
{\left[A^{\mu}\right]_{j} } & =\left[M_{0}^{-1} \partial^{\mu} \mathcal{V}\right]_{j}+\frac{1}{m_{j}} \epsilon^{\mu \nu} \partial_{\nu}\left([\mathcal{C}]_{j}-[\mathcal{B}]_{j}\right)
\end{aligned}
$$

The relations (4.5) and (4.6) do not immediately allow us to write down the correlation functions because, as in section $3, M_{0}^{2}$ may be complicated, but we can write ${ }^{5}$

$$
M_{0}^{2}=M^{2}-\frac{1}{\pi} e e^{T}=M\left(I-\frac{1}{\pi} M^{-1} e I_{f} e^{T} M^{-1}\right) M
$$

\footnotetext{
${ }^{5}$ The $I_{f}$ is unnecessary, but it is included to make the rest of the notation below more transparent.
} 
where $I_{f}$ is the identity matrix in the $n_{F}$ dimensional space of the fermions. We should be able to find an inverse of the form

$$
M_{0}^{-2}=M^{-1}\left(I+\frac{1}{\pi} M^{-1} e G_{f} e^{T} M^{-1}\right) M^{-1}
$$

Where $G_{f}$ is an $n_{F} \times n_{F}$ matrix. What we need is (and this should remind you of (3.23))

$$
\begin{aligned}
M_{0}^{2} M_{0}^{-2}=I & =M\left(I+\frac{1}{\pi} M^{-1} e\left(G_{f}-I_{f}-\frac{1}{\pi} e^{T} M^{-2} e G_{f}\right) e^{T} M^{-1}\right) M^{-1} \\
& =M\left(I+\frac{1}{\pi} M^{-1} e\left(G_{f}-I_{f}-B_{f} G_{f}\right) e^{T} M^{-1}\right) M^{-1}
\end{aligned}
$$

which is satisfied if

$$
G_{f}=\left(I_{f}-B_{f}\right)^{-1}
$$

where the symmetric $n_{F} \times n_{F}$ matrix $B_{f}$ is defined by

$$
B_{f} \equiv \frac{1}{\pi} e^{T} M^{-2} e
$$

The matrix $M_{0}^{2}$ will be positive as long as the eigenvalues of the matrix

$$
\tilde{B}_{f} \equiv \frac{1}{\pi} M^{-1} e e^{T} M^{-1}
$$

are less than one. When the largest eigenvalue goes to one, we go to the analog of the Schwinger point.

$$
\frac{1}{\pi} M^{-1} e e^{T} M^{-1} v=v
$$

Acting on the left of both sides with $e^{T} M^{-1}$ gives

$$
B_{f} e^{T} M^{-1} v=e^{T} M^{-1} v
$$

This correspondence holds for all non-zero eigenvalues. If there is a non-zero $v$ for which

$$
\frac{1}{\pi} M^{-1} e e^{T} M^{-1} u=\lambda u
$$

with $\lambda \neq 0$, then $e^{T} M^{-1} u$ cannot vanish (because that would imply $\lambda=0$ ) so that acting on the left of both sides with $e^{T} M^{-1}$ gives

$$
B_{f} e^{T} M^{-1} u=\lambda e^{T} M^{-1} u
$$

Thus very non-zero eigenvalue of $M^{-1} e e^{T} M^{-1}$ is an eigenvalue of $B_{f}$. A similar argument shows that every non-zero eigenvalue of $B_{f}$ is an eigenvalue of $M^{-1} e e^{T} M^{-1}$.

When we use (4.5), (4.6), (4.8), and (4.10) to write down the correlation functions, some useful simplifications occur. As we note in section 3 , the $\mathcal{V}$ and $\mathcal{C}$ propagators are proportional to the identity in the gauge boson space. The implies, for example, that $M_{0}^{-1}$ appears in the fermion correlators only in the combination

$$
\begin{aligned}
e^{T} M_{0}^{-2} e & =e^{T} M^{-2} e+\frac{1}{\pi} e^{T} M^{-2} e G_{f} e^{T} M^{-2} e \\
& =\pi\left(B_{f}+B_{f}\left(I-B_{f}\right)^{-1} B_{f}\right)=\pi B_{f}\left(I-B_{f}\right)^{-1}
\end{aligned}
$$


In correlators involving a single $A_{j}^{\mu}$, a similar simplifcation obtains because the $e^{T} \cdot M_{0}^{-1}$ in (4.5) is multiplied by and $M_{0}^{-1}$ from (4.6) and correlator involves only the combination

$$
\begin{aligned}
e^{T} M_{0}^{-2} & =e^{T} M^{-2} e+\frac{1}{\pi} e^{T} M^{-2} e G_{f} e^{T} M^{-2} \\
& =\pi\left(B_{f}+B_{f}\left(I-B_{f}\right)^{-1} B_{f}\right)=\pi\left(I-B_{f}\right)^{-1} e^{T}
\end{aligned}
$$

\section{Correlation functions}

In the notation of section 4, the fermion correlation functions in generalized Sommerfield model with $n_{A}$ vector bosons and $n_{F}$ fermions to infinite order in perturbation theory are written explicitly below. The most general fermion correlation function involves $n_{1 \alpha}$ left-handed fermion and anti-fermions of type $\alpha, \psi_{\alpha 1}\left(x_{1 \alpha j}\right)$ and $\psi_{\alpha 1}^{*}\left(y_{1 \alpha j}\right)$ where $j=1$ to $n_{1 \alpha}$ and $n_{2 \alpha}$ right-handed fermions and anti-fermions of type $\alpha, \psi_{\alpha 2}\left(x_{2 \alpha j}\right)$ and $\psi_{\alpha 2}^{*}\left(y_{2 \alpha j}\right)$ where $j=1$ to $n_{2 \alpha}$. We find

$$
\begin{aligned}
& \left\langle 0\left|T\left(\prod_{\alpha=1}^{n_{F}} \prod_{j=1}^{n_{1 \alpha}} \psi_{\alpha 1}\left(x_{1 \alpha j}\right) \psi_{\alpha 1}^{*}\left(y_{1 \alpha j}\right)\right)\left(\prod_{\alpha=1}^{n_{F}} \prod_{k=1}^{n_{2 \alpha}} \psi_{\alpha 2}\left(x_{2 \alpha k}\right) \psi_{\alpha 2}^{*}\left(y_{2 \alpha k}\right)\right)\right| 0\right\rangle \\
& =\left(\prod_{\alpha=1}^{n_{F}} i^{n_{1 \alpha}+n_{2 \alpha}}(-1)^{\frac{n_{1 \alpha}\left(n_{1 \alpha}-1\right)+n_{2 \alpha}\left(n_{2 \alpha}-1\right)}{2}}\right) \\
& \times\left(\prod_{\alpha=1}^{n_{F}} \prod_{\beta=1}^{n_{F}} \prod_{j=1}^{n_{1 \alpha}} \prod_{k=1}^{n_{1 \beta}} C_{0}^{\alpha \beta}\left(x_{1 \alpha j}-y_{1 \beta k}\right) C^{\alpha \beta}\left(x_{1 \alpha j}-y_{1 \beta k}\right) S_{1}^{\alpha \beta}\left(x_{1 \alpha j}-y_{1 \beta k}\right)\right) \\
& \times\left(\prod_{\alpha=1}^{n_{F}} \prod_{\beta=1}^{n_{F}} \prod_{j=1}^{n_{2 \alpha}} \prod_{k=1}^{n_{2 \beta}} C_{0}^{\alpha \beta}\left(x_{2 \alpha j}-y_{2 \beta k}\right) C^{\alpha \beta}\left(x_{2 \alpha j}-y_{2 \beta k}\right) S_{2}^{\alpha \beta}\left(x_{2 \alpha j}-y_{2 \beta k}\right)\right) \\
& \times\left(\prod_{\substack{\alpha \leq \beta, j<k \\
\text { for } \alpha=\beta}} C_{0}^{\alpha \beta}\left(x_{1 \alpha j}-x_{1 \beta k}\right)^{-1} C^{\alpha \beta}\left(x_{1 \alpha j}-x_{1 \beta k}\right)^{-1} S_{1}^{\alpha \beta}\left(x_{1 \alpha j}-x_{1 \beta k}\right)^{-1}\right) \\
& \times\left(\prod_{\substack{\alpha \leq \beta, j<k \\
\text { for } \alpha=\beta}} C_{0}^{\alpha \beta}\left(x_{2 \alpha j}-x_{2 \beta k}\right)^{-1} C^{\alpha \beta}\left(x_{2 \alpha j}-x_{2 \beta k}\right)^{-1} S_{2}^{\alpha \beta}\left(x_{2 \alpha j}-x_{2 \beta k}\right)^{-1}\right) \\
& \times\left(\prod_{\substack{\alpha \leq \beta, j<k \\
\text { for } \alpha=\beta}} C_{0}^{\alpha \beta}\left(y_{1 \alpha j}-y_{1 \beta k}\right)^{-1} C^{\alpha \beta}\left(y_{1 \alpha j}-y_{1 \beta k}\right)^{-1} S_{1}^{\alpha \beta}\left(y_{1 \alpha j}-y_{1 \beta k}\right)^{-1}\right) \\
& \times\left(\prod_{\substack{\alpha \leq \beta, j<k \\
\text { for } \alpha=\beta}} C_{0}^{\alpha \beta}\left(y_{2 \alpha j}-y_{2 \beta k}\right)^{-1} C^{\alpha \beta}\left(y_{2 \alpha j}-y_{2 \beta k}\right)^{-1} S_{2}^{\alpha \beta}\left(y_{2 \alpha j}-y_{2 \beta k}\right)^{-1}\right) \\
& \times\left(\prod_{\alpha=1}^{n_{F}} \prod_{\beta=1}^{n_{F}} \prod_{j=1}^{n_{1 \alpha}} \prod_{k=1}^{n_{2 \beta}} C_{0}^{\alpha \beta}\left(x_{1 \alpha j}-y_{2 \beta k}\right) C^{\alpha \beta}\left(x_{1 \alpha j}-y_{2 \beta k}\right)^{-1}\right) \\
& \times\left(\prod_{\alpha=1}^{n_{F}} \prod_{\beta=1}^{n_{F}} \prod_{j=1}^{n_{2 \alpha}} \prod_{k=1}^{n_{1 \beta}} C_{0}^{\alpha \beta}\left(x_{2 \alpha j}-y_{1 \beta k}\right) C^{\alpha \beta}\left(x_{2 \alpha j}-y_{1 \beta k}\right)^{-1}\right)
\end{aligned}
$$




$$
\begin{aligned}
& \times\left(\prod_{\alpha=1}^{n_{F}} \prod_{\beta=1}^{n_{F}} \prod_{j=1}^{n_{1 \alpha}} \prod_{k=1}^{n_{2 \beta}} C_{0}^{\alpha \beta}\left(x_{1 \alpha j}-x_{2 \beta k}\right)^{-1} C^{\alpha \beta}\left(x_{1 \alpha j}-x_{2 \beta k}\right)\right) \\
& \times\left(\prod_{\alpha=1}^{n_{F}} \prod_{\beta=1}^{n_{F}} \prod_{j=1}^{n_{1 \alpha}} \prod_{k=1}^{n_{2 \beta}} C_{0}^{\alpha \beta}\left(y_{1 \alpha j}-y_{2 \beta k}\right)^{-1} C^{\alpha \beta}\left(y_{1 \alpha j}-y_{\beta y 2 k}\right)\right)
\end{aligned}
$$

where for the Sommerfield model

$$
\begin{aligned}
& C^{\alpha \beta}(x)=\exp \left[\sum_{j=1}^{n_{A}} \frac{e_{j \alpha} e_{j \beta}}{2 \pi m_{j}^{2}}\left[K_{0}\left(m_{j} \sqrt{-x^{2}+i \epsilon}\right)+\ln \left(\xi m_{j} \sqrt{-x^{2}+i \epsilon}\right)\right]\right] \\
& C_{0}^{\alpha \beta}(x)=\exp \left[i \pi\left[B_{f}\left(I-B_{f}\right)^{-1}\right]_{\alpha \beta}[D(x)-D(0)]\right] \propto\left(-x^{2}+i \epsilon\right)^{-\left[B_{f}\left(I-B_{f}\right)^{-1}\right]_{\alpha \beta} / 4}
\end{aligned}
$$

with

$$
\xi=\frac{e^{\gamma_{E}}}{2}
$$

and where the matrix $B_{f}$ from (4.11) can be written as

$$
\begin{aligned}
{\left[B_{f}\right]_{\alpha \beta} } & =\sum_{j} \frac{e_{j \alpha} e_{j \beta}}{\pi m_{j}^{2}} \\
S_{1}^{\alpha \beta}(x) & = \begin{cases}S_{1}(x) & \text { if } \alpha=\beta \\
1 & \text { if } \alpha \neq \beta\end{cases} \\
S_{1}(x) & =\int \frac{d^{2} p}{(2 \pi)^{2}} e^{-i p x} \frac{p^{0}+p^{1}}{p^{2}+i \epsilon}=-\frac{1}{2 \pi} \frac{x^{0}+x^{1}}{x^{2}-i \epsilon} \\
S_{2}^{\alpha \beta}(x) & = \begin{cases}S_{2}(x) & \text { if } \alpha=\beta \\
1 & \text { if } \alpha \neq \beta\end{cases} \\
S_{2}(x) & =\int \frac{d^{2} p}{(2 \pi)^{2}} e^{-i p x} \frac{p^{0}-p^{1}}{p^{2}+i \epsilon}=-\frac{1}{2 \pi} \frac{x^{0}-x^{1}}{x^{2}-i \epsilon}
\end{aligned}
$$

The ten contributions in (5.3)-(5.12) correspond to the ten lines in the diagram in figure 1. Eq. (5.2) includes a factor of $i$ for every free-fermion correlator and factors of $(-1)^{n(n-1) / 2}$ from combining the different contractions of left-handed and right-handed free-fermions into a single factor.

\section{Conclusions}

The generalizations of the Sommerfield model analyzed here are a rich vein of exactly solvable quantum field theories some of which can exhibit properties that are very different from the those of the simple Sommerfield and Schwinger models. We look forward to mining this lode in future work.

\section{Acknowledgments}

This work is supported in part by NSF grant PHY-1719924. BW's research is supported in part by the Harvard College Research Program. 


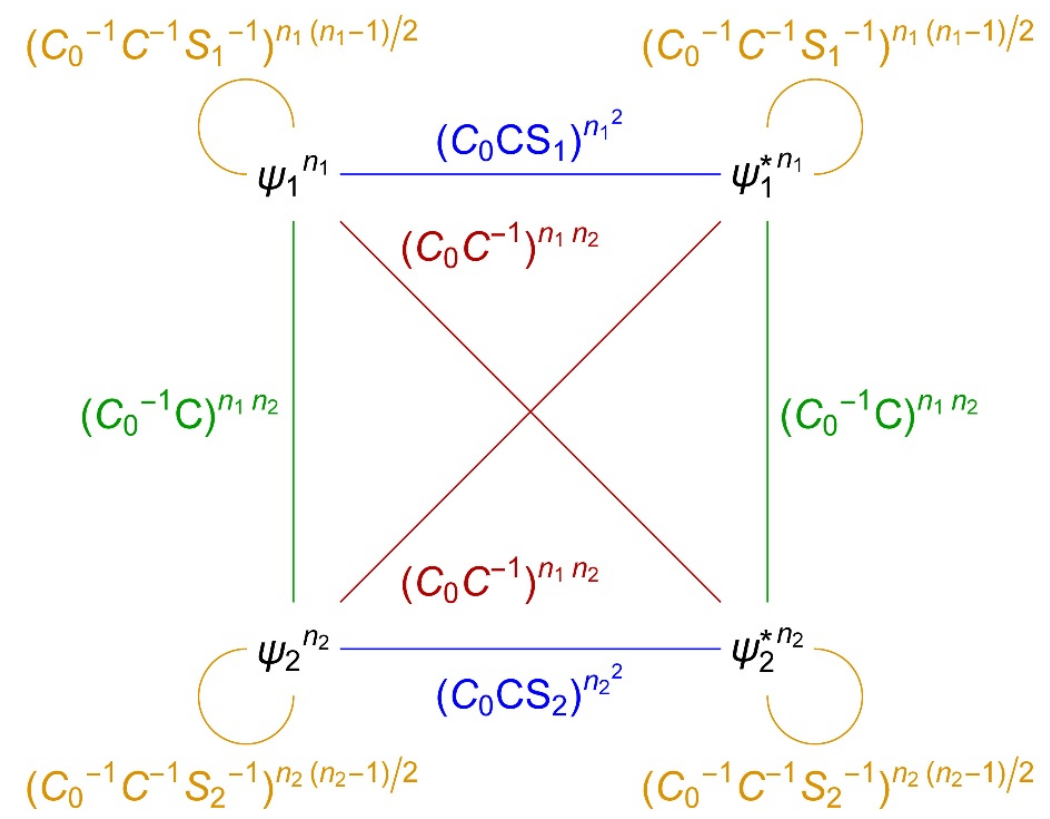

Figure 1. Pictorial representation of the fermion correlation functions.

Open Access. This article is distributed under the terms of the Creative Commons Attribution License (CC-BY 4.0), which permits any use, distribution and reproduction in any medium, provided the original author(s) and source are credited.

\section{References}

[1] J.S. Schwinger, Gauge invariance and mass. II, Phys. Rev. 128 (1962) 2425 [INSPIRE].

[2] K.G. Wilson, Nonlagrangian models of current algebra, Phys. Rev. 179 (1969) 1499 [INSPIRE].

[3] S.R. Coleman, More about the massive Schwinger model, Annals Phys. 101 (1976) 239 [INSPIRE].

[4] C.M. Sommerfield, On the definition of currents and the action principle in field theories of one spatial dimension, Ann. Phys. 26 (1964) 1.

[5] H. Georgi and Y. Kats, An unparticle example in 2D, Phys. Rev. Lett. 101 (2008) 131603 [arXiv:0805.3953] [INSPIRE].

[6] J.H. Lowenstein and J.A. Swieca, Quantum electrodynamics in two-dimensions, Annals Phys. 68 (1971) 172 [INSPIRE].

[7] K.D. Rothe and J.A. Swieca, Gauge transformations and vacuum structure in the Schwinger model, Phys. Rev. D 15 (1977) 541 [INSPIRE].

[8] L.V. Belvedere et al., Generalized two-dimensional abelian gauge theories and confinement, Nucl. Phys. B 153 (1979) 112 [INSPIRE].

[9] H. Georgi, The Schwinger point, JHEP 11 (2019) 057 [arXiv: 1905.09632] [INSPIRE]. 
[10] H. Georgi and B. Warner, Non-perturbative effects in generalized Schwinger models, arXiv: 1908.03279.

[11] H. Georgi and Y. Kats, Unparticle self-interactions, JHEP 02 (2010) 065 [arXiv:0904.1962] [INSPIRE].

[12] K.G. Wilson, Operator product expansions and anomalous dimensions in the Thirring model, Phys. Rev. D 2 (1970) 1473 [inSPIRE].

[13] R. Roskies and F. Schaposnik, Comment on Fujikawa's analysis applied to the Schwinger model, Phys. Rev. D 23 (1981) 558 [InSPIRE].

[14] H. Georgi and J.M. Rawls, Anomalies of the axial-vector current in two dimensions, Phys. Rev. D 3 (1971) 874 [INSPIRE]. 